\title{
Cholera outbreak investigation in four districts of Kirkos sub-city in Addis Ababa, Ethiopia: A case-control study
}

\author{
Tamiru Tadesse ${ }^{1 *}$ and Belay Zawdie ${ }^{2}$ \\ ${ }^{1}$ Border and Travel Health Service Directorate, Ethiopian Public Health Institute, Ethiopia \\ ${ }^{2}$ School of Biomedical Sciences, Jimma University, Ethiopia
}

\begin{abstract}
Background: Cholera is becoming a big problem in the world especially in African region including Ethiopia. The disease is very common in areas where there is inequity and lack of social development. The diseases affected the whole districts of the kirkos-sub-city, Addis Ababa. Hence, we are enforced to assess risk factors associated with cholera.

Methods: Unmatched 1:2 case-control study on 50 confirmed cases and 100 controls was conducted from June 09 , 2016 to September 2016 . Data were collected through direct interviews using semi-structured and pre-tested questionnaires. Two data collectors and one supervisor were involved in data collection. Cases were selected from cholera treatment center line list and controls were selected from neighborhood of case using lottery method. Data were entered by Epi Info and analyzed using SPSS version 21 software. Logistic regression was used to compute the crude and adjusted odds ratios for the factors associated with acquiring the AWD. A p-value of $<0.05$ at $95 \%$ CI was considered to be statistically significant.

Results: The median and mode age in the study groups was 35.5 and 60 years old respectively with interquartile range from 28 to 54.25 years. The highest peak period for the outbreak was on July 4, 2016. Eating partially roasted meat [AOR=4.14, CI=1.11-15.46] and being male [AOR=8.57, CI=2.21-33.25] had significantly associated with the risk factors of accruing cholera. Whereas, regular hand washing with soap after defecation $[\mathrm{AOR}=0.23$, CI=0.06-0.91], treating water before drinking by aqua tabs $[\mathrm{AOR}=0.08, \mathrm{CI}=0.01-0.95]$ and boiling $[\mathrm{AOR}=0.23, \mathrm{CI}=0.06-0.95]$ disposing house hold refuse at municipal site $[\mathrm{AOR}=0.11, \mathrm{CI}=0.02-$ 0.69] were protective against cholera.

Conclusions: Eating partially roasted meat, regular hand washing with soap after defecation, disposing house hold refuse at municipal site, treating water before drinking by aqua tabs and boiling were possible risk factors associated with the outbreak. Hence, water, sanitation and hygiene offices should strictly work on the hygiene and availability of safe water at all levels.
\end{abstract}

\begin{abstract}
Abbreviations: AR-Attack Rate, AOR: Adjusted Odd Ratio, CFR: Case Fatality Rate, CI: Confidence Interval, COR: Crude Odd ratio, CTC : Cholera Treatment Center, EFY: Ethiopian Fiscal Year, EPHI: Ethiopia Public Health Institute, Gov't: Government, HDA : Health Development army, HH: House Hold, MoH: Ministry of Health, NGO: Non-Governmental Organization, OPD: Out Patient Department, RRT: Rapid Response Team, TGL-Treatment Guide Line, US: United State, UHEW: Urban Health Extension Worker, WASH-Water and Sanitation hygiene, WHO: World Health Organization
\end{abstract}

\section{Background}

Cholera is a diarrheal disease caused by infection of the intestine with the bacterium Vibrio cholerae, either type O1 or O139; usually transmitted through faecally contaminated water or food and remains an ever-present risk in many countries. It becomes also a global threat to public health and a key indicator of inequity and lack of social development. In 2008 alone, a total of 190,130 cases were notified from 56 countries, including 5143 deaths(1). Many more cases were unaccounted for due to limitations in surveillance systems and fear of trade and travel sanctions. The true burden of the disease is estimated to be 1.3 to 4.0 million cases and 21000 to 143000 death worldwide annually due to cholera [1]. In2007, various countries around the world notified 178677 cases of cholera and 4033 cholera deaths to the World Health Organization [2]. About, $62 \%(110,837)$, of the global burden of cholera cases and $56.7 \%$ of death were notified to WHO from African Region alone which resulted in huge economic loss with millions of US\$ in different life expectancies meaning US $\$ 43.3$ million, US\$60 million and US\$72.7 million, assuming life expectancies of 40, 53 and 73 years respectively [2]. Globally, the number of deaths from cholera rose from 4948 in 2009 to 7543 in 2010, an increase of 52\% with an overall CFR of $2.38 \%$. Of the 32 countries that reported deaths from cholera, 20 were on the African continent: these countries accounted for 3397 deaths and $45 \%$ of the global total [3]. The number of cholera cases reported to $\mathrm{WHO}$ has continued to be high over the last few years. During 2015,172 454 cases were notified from 42 countries, including 1304 deaths [4].

In Ethiopia it was indicated that, there was cholera epidemic in 1990 which persisted with recrudescence of cases till 1998 [5]. Moreover, from July 2008 to June 2009 in Ethiopia, there were a total

${ }^{\star}$ Correspondence to: Tamiru Tadesse, Border and Travel Health Service Directorate, Addis Ababa, Ethiopian Public Health Institute, Ethiopia, E-mail: tamirutadesse@yahoo.com

Key words: cholera, outbreak, risk factors, kirkos sub-city, addis ababa, Ethiopia

Received: December 05, 2019; Accepted: December 21, 2019; Published: December 23, 2019 
of 9485 cases and 193 deaths (with case-fatality rate $=2.0 \%$ ) of cholera in 70 Woredas of seven regions and one urban administration; Addis Ababa city administration. The Regional distribution shows that the highest number of cases 2,988(31.5\%) occurred in Afar, followed by Oromia 2,272(23.9\%) and least in Addis Ababa 12[0.13\%] [6].

Currently a number of cholera cases were seen in kirkos sub-city following a case seen at kolfe keranio sub-city and thus, the subcity experienced cholera outbreak declared on 8 June, 2016 and was laboratory confirmed by culture on 7 June, 2016 by EPHI. Accordingly, our team ,surveillance team, joins other teams organized at kirkos subcity that consists of different health professional (Physicians, nurses, Epidemiologists, Public health \&Environmental Health professionals) and were organized and deployed to the site for the assessment of the outbreak (response and control) and to provide technical support in the control of the outbreak. These teams also activate rapid response team (RRT) at all districts of the sub-city as per cholera outbreak management guideline. These teams are categorized into active search and disinfection team, social mobilization (social awareness) team, WASH team and supply/logistic management team.

Hence, Unmatched Case control study was conducted to support the outbreak response through identifying associated risk factors in order to generate evidence based decision in four districts of kirkos sub-city from June 9 to September 13, 2016.Selection of the district was just for simplicity issues however, responses of the outbreak were done in all affected districts of the sub-city.

\section{Methods}

\section{Study areas and period}

The outbreak investigation was conducted in four districts of kirkos sub-city, Addis Ababa city administration from June 09 to September 2016. The population of the districts was $30573,15642,34818$ and 27971 in 02, 03, 04 and 11 districts respectively for 2016.

Kirkos sub-city is one of the 10 sub-cities in Addis Ababa city administration which is located at the center of city; Kazanchis bounded by Arada and Yeka in North, Bole in East, Lideta in West and Nifas Silk Lafto and Bole in South direction. Kirkos Sub-city has a Subtropical highland climate [7] which varies from seasons of summer, 9 months to cool months of rainfall, about three months, with an overall average maximum and minimum temperature of 22.9 and 10.8 degree centigrade, respectively. Kirkos sub-city has 11 Woreda and 41 SubWoreda, former kebele.

For cholera outbreak, kirkos sub-city has been considered to be virgin sub-city for more than six years ago as of all sub-cities in Addis Ababa city administration. However, Kirkos sub-city has experienced cholera outbreak on 8 June, 2016 which was confirmed by culture on 7 June, 2016 by EPHI.

\section{Study design}

Community based unmatched 1:2 case-control study was conducted in four districts of kirkos Sub-city Addis Ababa, Ethiopia.

\section{Sample size determination}

Sample size was calculated by StatCalc Epi Info version 7 by the following assumption(8).

Least extreme Odds ratio to be detected $=0.2$

Proportion of Exposed controls $=29 \%$
Proportion of Exposed cases $=15 \%$

Confidence level $=95 \%$, Power $=80 \%$

Number of cases $=46$, Number of controls $=92$

Total sample size $=138($ Fleiss $\mathrm{w} / \mathrm{cc}$ et $\mathrm{al})$

However, since it was an emergency situation, we assumed a sample size of 50 RDT confirmed cases and 100 controls with 1:2 ratios.

\section{Appendix}

Case: Person who is newly confirmed cases of cholera admitted into the cholera treatment center.

Control: Person living in the same neighborhood of a case but who had not suffered cholera until the date of the interview. When there are more than two eligible a lottery method was used to select appropriate control for each case.

\section{Inclusion and exclusion criteria}

\section{Inclusion criteria}

For cases, a person who are newly confirmed of cholera and admitted into the cholera treatment center and for controls, a person living in the same neighborhood as of a case but who had not suffered cholera until the date of the interview was included in the study groups.

\section{Exclusion criteria}

Person who is not able to respond and communicate to our interview and children less than five years old were also excluded from the study groups.

\section{Data collection instrument and procedures}

Data were collected by using a semi-structured questionnaire. A semi-structured face to face interview questionnaire was prepared from cholera outbreak management guide which was prepared by Ethiopian public health institutes for the cholera outbreak risk assessment [9]. We also include pertinent questionnaire from another similar setup [10]. The questionnaire prepared by English was translated into Amharic and back to English by lingual experts for checking correctness. Finally, Amharic translated questionnaire was provided to the study participants by two trained urban health extension.

\section{Data quality management}

Data collection tools were adapted from other literatures and prepared by a principal investigator. A semi-structured questionnaire was translated into Amharic language and back-translated to the English language to maintain its consistency and corrective. Training was given for the data collectors and field supervisors on the objective of the study, contents of the questionnaire and how to maintain confidentiality and privacy of the participants. Finally, pre-test was performed at $5 \%$ of the sample size in other districts of kirkos subcity before the actual data collection. Data collection tools were revised, edited and modified according to the results of the pre-test. A principal investigator and field supervisor had checked collected data for their completeness on the daily basis before the data were entered into the software. More orientation for those data collectors come up with incompleteness and inconsistency was provided in between data collecting period. In the case of the incompleteness and inconsistency, the collected data were discarded as incomplete. 


\section{Statistical analyses}

Interviewed data were cleaned and entered into EPI info7 version software and analyzed by SPSS window version 21 . Bivariate and Multivariable analysis was applied using binary logistic regression to identify associated factors with acquiring the cholera in the district. Variables at P-value less than 0.05 at $95 \%$ CI were considered as significant factors for the study participant in acquiring the cholera.

\section{Result}

\section{Descriptive epidemiology}

A total of 156 confirmed cholera cases and zero deaths were registered during June 9 to Sept. 13, 2016 in the four districts of the sub-city with an attack rate (AR) and case fatality rate (CFR) of $0.143 \%$ and $0 \%$ respectively. Among all cases in four districts, 94 (60.25\%) were males and 62 (65.9\%) were in the age category of $15-44$ years old. The median interval age was also in between $15-44$ years old. The highest proportion of cases, 60 (38.4\%), was seen in 11 district followed by 04 district with 54 (34.6\%) cases (Table 1).

In 02 district, the onset date of the first cases was recorded on June 17, 2016. From this date to Sept.8, 2016, 20 cases and 0 deaths were reviewed from kirkos sub-city CTC line list registry with a $0.065 \mathrm{AR} \%$ and case fatality rate of $0 \%$. Males and the age category $15-44$ years old constituted 17 (85\%) and 12 (60\%) of the cases respectively. Most cases, $12(60 \%)$, was in the age category of $15-44$ years old and the median age in this district was 36.5 years old. All kebeles and ketenas in the district were affected by cholera.

In 03 districts the onset date of the first case was on June16, 2016. From this date to Sept.11, 2016, 22 cases and zero deaths were reviewed from the kirkos sub-city CTC line list registry which had a zero case fatality rate Similar to other three districts and $0.140 \%$ AR. Most of the cases, $18(81.8 \%)$, were males and $17(77.27 \%)$ of the cases were in the age range of 15-44yers old with median of 29.5 years old. All kebele's/ ketena's in the district were affected by cholera.

In 04 districts the onset date of the first case was on June15, 2016. From this date to August 18, 2016, 54 cases and zero death were reviewed from kirkos CTC line list registry which also had zero case fatality rate similar to other three districts and $0.155 \%$ AR. Most cases, $28(51.8 \%)$, were females and $31(57.4 \%)$ of the cases were in the age range of 15-44 years old with the median of 38.5 years old. All kebeles/ ketenas of the district were affected by cholera.

In 11 districts the onset of the first case was on June15, 2016. From this date to Sept.10, 2016, 60 cases and zero death were reviewed from kirkos CTC line list registry which had zero case fatality similar to other three districts and $0.214 \%$ AR. This district shows the highest proportion of the case compared to the other three districts listed above. Most cases, 33(55\%), were males and 32(53.3\%) of the cases were in the age range of $15-44$ years old with the median of 38.5 years old as of in 04 district. All Kebeles and ketenas in this district were affected by cholera.

\section{Stool specimen testing result}

Stool samples were collected from the first two cases of cholera and were confirmed by culture on June 7, 2016 at Ethiopian public health institute which is enough for outbreak situation [11] and found to be positive for Cholera Enaba O1.

The epidemic was started on june8, 2016 as the whole in Addis Ababa city administration following cases reported from kolfe keranio sub-city. The first case was reported in Kirkos Sub-city district 10, particularly from Kebele $44 / 45$ on June 09,2016 . Hence,the sub-city experienced the outbreak on June 09,2016. As shown in Epidemic curve the peak of the outbreak was on July 4, 2016 (Figure 1).

\section{Water and food sample testing}

Water samples from different areas of tap water source, holy water, and food samples (which include raw meat and locally made beverage) from different food establishment and swabs from different butcher and slaughter houses were taken and analyzed at Ethiopian public health institute and found to be contaminated with cholera Enba O1.

\section{Analytic Epidemiology}

A total of 50 cases and 100 controls were recruited in the study from four districts of the kirkos sub-city located in the city government of Addis Ababa. Responses were received from all study participants indicating a response rate of $100 \%$ (Table 2).

Table 1. Case distribution by sex and attack rate in four districts of kirkos sub-city, 2016

\begin{tabular}{|c|c|c|c|c|c|c|}
\hline \multirow{2}{*}{ District } & \multirow{2}{*}{$\begin{array}{c}\text { Total population for } \\
2016\end{array}$} & \multicolumn{2}{|c|}{ Sex } & \multirow{2}{*}{ Total } & \multirow{2}{*}{$\%$} & \multirow{2}{*}{ AR\% } \\
\hline & & Female & Male & & & \\
\hline 02 & 30569 & 3 & 17 & 20 & 13 & 0.0654 \\
\hline 03 & 15635 & 4 & 18 & 22 & 14 & 0.1407 \\
\hline 04 & 34812 & 28 & 26 & 54 & 35 & 0.1551 \\
\hline 11 & 27967 & 27 & 33 & 60 & 38 & 0.2145 \\
\hline Total & 108,983 & 62 & 94 & 156 & 100 & 0.143 \\
\hline
\end{tabular}

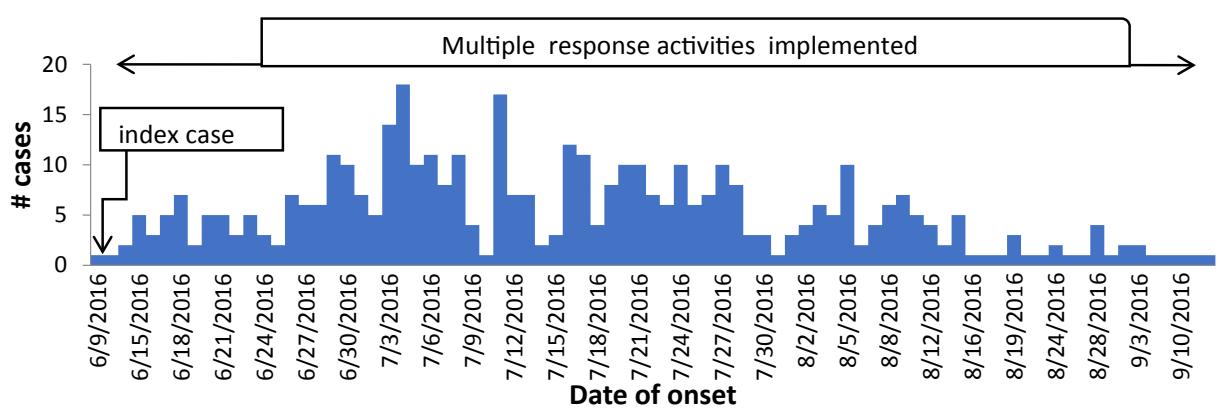

Figure 1. Epidemic Curve, Kirkos sub-city, June 9 to sept.13, 2016 
Table 2. Demographic characteristics of the cases and control ( $\mathrm{n}=150)$, Kirkos sub-city, 2016

\begin{tabular}{|c|c|c|c|}
\hline \multicolumn{2}{|c|}{ Descriptive variables } & \multirow{2}{*}{$\begin{array}{c}\text { Case }(\mathbf{n}=50) \\
\text { No. }(\%) \\
29(58)\end{array}$} & \multirow{2}{*}{$\begin{array}{c}\begin{array}{c}\text { Control (n=100) } \\
\text { No. }(\mathbf{\%})\end{array} \\
16(16) \\
\end{array}$} \\
\hline Sex & Male & & \\
\hline sex & Female & $21(42)$ & $84(84)$ \\
\hline \multirow{4}{*}{ District } & 02 & $5(10)$ & $11(11)$ \\
\hline & 03 & $4(8)$ & $29(29)$ \\
\hline & 04 & $17(34)$ & $31(31)$ \\
\hline & 11 & $24(48)$ & $29(29)$ \\
\hline \multirow{3}{*}{ Age group in years } & $5-14$ & $3(6)$ & $2(2)$ \\
\hline & $15-44$ & $28(56)$ & $66(66)$ \\
\hline & $\geq 45$ & $19(38)$ & $32(32)$ \\
\hline \multirow{9}{*}{ Occupation } & Daily laborer & $7(14)$ & $13(13)$ \\
\hline & Gov't employee & $4(8)$ & $34(34)$ \\
\hline & House wife and homemade servant & $2(4)$ & $1(1)$ \\
\hline & Janitor & $0(0)$ & $2(2)$ \\
\hline & NGO & $15(30)$ & $23(23)$ \\
\hline & Private employee & $6(12)$ & $12(12)$ \\
\hline & Retired & $4(8)$ & $8(8)$ \\
\hline & Student & $5(10)$ & $6(6)$ \\
\hline & Unemployed & $7(14)$ & $1(1)$ \\
\hline
\end{tabular}

Table 3. Bivariate and Multivariable analysis for risk factor associated with cholera in four districts of kirkos sub-city, 2016

\begin{tabular}{|c|c|c|c|c|c|c|c|c|}
\hline \multirow[b]{2}{*}{ Risk factors } & \multirow{2}{*}{$\begin{array}{c}\text { Case }(n=50) \\
\text { Yes } \\
\text { No }(\%)\end{array}$} & \multirow{2}{*}{$\begin{array}{c}\text { Control } \\
(n=100) \\
\text { Yes } \\
\text { No }(\%)\end{array}$} & \multicolumn{3}{|c|}{ Bivariate analysis } & \multicolumn{3}{|c|}{ Multivariable analysis } \\
\hline & & & COR & $95 \% \mathrm{CI}$ & p-value & AOR & $95 \%$ CI & p-value \\
\hline Sex (male) & $29(58)$ & $16(16)$ & 7.25 & $3.34-15.74$ & 0.000 & 8.57 & $2.21-33.25$ & $0.002 *$ \\
\hline \multicolumn{9}{|l|}{$\begin{array}{l}\text { Water treatment before } \\
\text { drinking by; }\end{array}$} \\
\hline - Aqua tabs & $1(2)$ & $24(24)$ & 0.07 & $0.01-0.49$ & 0.008 & 0.08 & $0.01-0.95$ & $0.045^{*}$ \\
\hline - Boiling & $7(14)$ & $41(41)$ & 0.23 & $0.10-0.57$ & 0.001 & 0.23 & $0.06-0.95$ & $0.042^{*}$ \\
\hline \multicolumn{9}{|l|}{ Food exposure } \\
\hline $\begin{array}{l}\text { - Eating partially roasted } \\
\text { meat }\end{array}$ & $30(60)$ & $26(26)$ & 4.27 & $2.08-8.78$ & 0.000 & 4.14 & $1.11-15.46$ & $0.034^{*}$ \\
\hline - Eating fruits & $18(36)$ & 19(19) & 2.40 & $1.12-5.15$ & 0.025 & 3.73 & $0.97-14.40$ & 0.056 \\
\hline $\begin{array}{l}\text { - Drinking locally made } \\
\text { Besso }\end{array}$ & $14(28)$ & $13(13)$ & 2.60 & $1.11-6.08$ & 0.027 & 0.44 & $0.09-2.17$ & 0.311 \\
\hline $\begin{array}{l}\text { Regular hand washing with } \\
\text { soap after defecation }\end{array}$ & $23(46)$ & $83(83)$ & 0.17 & $0.08-0.37$ & 0.000 & 0.23 & $0.06-0.91$ & $0.037 *$ \\
\hline $\begin{array}{l}\text { Regular hand washing with } \\
\text { soap before meal/preparing } \\
\text { food }\end{array}$ & $25(50)$ & $82(82)$ & 0.22 & $0.10-0.47$ & 0.000 & 0.49 & $0.11-2.20$ & 0.351 \\
\hline $\begin{array}{l}\text { Disposing } \mathrm{HH} \text { refuse at } \\
\text { municipal site }\end{array}$ & $4(8)$ & $45(45)$ & 0.11 & $0.04-0.32$ & 0.000 & 0.11 & $0.02-0.69$ & $0.019^{*}$ \\
\hline Eating food at restaurant & $13(26)$ & $9(9)$ & 3.55 & $1.40-9.02$ & 0.008 & 4.25 & $0.83-21.94$ & 0.084 \\
\hline $\begin{array}{l}\text { Buying foods for } \mathrm{HH} \text { from } \\
\text { local market }\end{array}$ & $48(96)$ & $84(84)$ & 4.57 & $1.01-20.74$ & 0.049 & 4.48 & $0.55-36.76$ & 0.162 \\
\hline
\end{tabular}

* Binary logistic regression

From a total of study participants, 50 cases and 100 controls, $70 \%$ were females. From both study groups the median age was 35.5 years with interquartile range (IQR) from 28 to 54.25 years. Similarly, from both study groups the mode age was 60 years old.

From 50 confirmed cases, $58 \%$ were males. Fifteen (30\%) of the cases and twenty three $(23 \%)$ of the controls were Non-governmental employee (Table 2). Government employee and NGO employee constitute $25 \%$ from the total respondents followed by daily laborers, which were $14 \%$ (Table 3 ).

Forty three (86\%) of cases and 51 (51\%) of controls used communal tap water for drinking purpose. Twenty (40\%) cases and 78(78\%) of controls were practicing treating water before drinking. From the casecontrol study subjects, 34 (68 \%) case and 29(29\%) control ate/drink foods out-side their home in the past five days .Twenty five (50\%) of the cases and $82(82 \%)$ controls wash their hands with soap before having any meal/preparing food and 23(46\%) of cases and 83(83\%) of controls practice hand washing with soap after defecation. Eleven (22\%) of cases and $27(27 \%)$ of controls drink water from holy water site. Majority of the cases $28(56 \%)$ were in the age category of $15-44$ years old. Regarding to the symptoms; $47(94 \%)$ of the cases had dehydration and vomiting, while $50(100 \%)$ of the cases had watery diarrhea.

\section{Risk factors for contracting cholera}

Statistically significant risk factors of contracting cholera in the study groups were: being male $[\mathrm{COR}=7.25,95 \% \mathrm{CI}(3.34-15.74)]$; eating partially roasted meat $[\mathrm{COR}=4.27,95 \%$ CI $(2.08-8.78)]$; eating fruit $[\mathrm{COR}=2.4,95 \% \mathrm{CI}(1.12-5.15)]$; drinking locally made 
besso $[\mathrm{COR}=2.60,95 \% \mathrm{CI}(1.11-6.08)]$; eating food at restaurant $[\mathrm{COR}=3.55,95 \% \mathrm{CI}(1.40-9.02)]$ and; buying food from local market for house hold consumption $\{\mathrm{COR}=4.57,95 \% \mathrm{CI}(1.01-20.74)]$ (Table 3$)$.

Statistically significant protective factors against contracting cholera were: treating water before drinking by aqua tab $[\mathrm{COR}=0.07$, 95\% $\mathrm{CI}(0.01-0.49)]$ and boiling method $[\mathrm{COR}=0.23,95 \% \mathrm{CI}(0.10-$ 0.57)];regular hand washing with soap after defecation[COR = $0.17,95 \% \mathrm{CI}(0.08-0.37)]$;regular hand washing with soap before meal or preparing food $\mathrm{COR}=0.22,95 \% \mathrm{CI}(0.10-0.43)]$ and disposing house hold refuse at municipal site $\{\mathrm{COR}=0.11,95 \% \mathrm{CI}(0.04-0.32)]$ (Table 3 ).

\section{Multivariate analysis}

Eating partially roasted meat $[\mathrm{AOR}=4.14, \mathrm{CI}=(1.11-15.46)]$ and being male $[\mathrm{AOR}=8.57, \mathrm{CI}=(2.21-33.25)]$ were an independent risk factors for contracting cholera. Whereas, regular hand washing with soap after defecation $[\mathrm{AOR}=0.23, \mathrm{CI}=(0.06-0.91)]$, treating water before drinking by aqua tabs $[\mathrm{AOR}=0.08, \mathrm{CI}=(0.01-0.95)]$ and boiling method $[\mathrm{AOR}=0.23, \mathrm{CI}=(0.06-0.95)]$; disposing house hold refuse at municipal site $[\mathrm{AOR}=0.11,95 \% \mathrm{CI}(0.02$ -

\section{Discussion}

In this outbreak investigation study, the onset date of the first case was on June15, 2016 in district 04 and 11 and on June 16 and 17, 2016 in district 03 and 02 respectively. The rapid spread from district 04 and 11 to district 03 and 02 was likely because the first case was seen in district 10 of the sub-city which is the boundary of district 04 and 11 . The other reason could be due to the frequent movement of residents and daily laborers from one district to the others since they are boundary of each other.

The highest numbers of cases (38\%,35\% and 14\%) were occurred in district 11,04, and 03 respectively (Table 1) in the four districts of the sub-city. This might be due to the infected river crossing the first two districts by which the community residing near side the river have easy access for the contact with the contaminated river water. The Epicurve has many peaks (Figure 1) which showed a progressive person to person transmission, this could be due to the poor sanitation facility and poor personal hygiene and may also be weak response activity of the districts epidemic task force.

The overall attack rate (AR) and case fatality rate (CFR) was $0.154 \%$ and $0 \%$ respectively in kirkos sub-city and $0.143 \%$ and $0 \%$ in the four districts (case-control study area). This value $(0.143 \%)$ is lower than figures reported in AWD outbreak in Afar-Ethiopia in 2014 [10]. The AR and CFR in Afar-Ethiopia was $0.9 \%$ and $4.4 \%$ respectively. Similarly when we compare overall AR of this outbreak with other African countries like Ghana it was also lower than figures reported in cholera outbreak in Ghana in 2016 [12]. However, AR of this assessment was greater than AR of cholera reported in Nigeria [13]. Similarly, CFR of this outbreak was lower than the figures reported in the above three countries cholera outbreak report including AfarEthiopia [10]. This figures (Zero percent CFR) could be due to the reason that all death occurred at referral hospital assigned for the subcity catchment area were not incorporated into the sub-city's' AWD line list and for the lower AR the reason could be due to more attention given to the outbreak (high community awareness to the outbreak and easy accessibility of health facility in the sub-city). Even-though, the reason for higher figures reported in Afar-AWD outbreak were explained as less attention given for employee working in the investor farm, shortage of medical supplies and poor case management in AWD $\mathrm{TC}$, for this report the reason could be the inverse of the reason stated in Afar-Ethiopia AWD outbreak [10]. Age and sex specific attack rate and case fatality rate could not be calculated due to lack of estimate population per sex in the districts but, the case control study showed that majority of the cases were in the age category of the 15-44 years old which could be due to the wide range of ages category in between the 15-44 years compared to other two age category. It also shows that the majority of the study participants $(70 \%)$ were female which could be due to the more female controls and female cases were interviewed especially while we select controls, females or house wife were present at their home in which most of the time the date of interview were on the working day.

Most cases in all districts of the study areas were males. This was likely due to the fact that most of the time males are using their meals outside their home which may expose them to this infection.

This study also showed that most cases were non- governmental employee followed by daily laborer and unemployed persons than others occupation. These may be due to the fact that there are many different NGOs located in Addis Ababa as well as in the sub-city. For the high composition of daily laborer may be due to their frequent movement from one district to the others to get daily job and their personal hygiene in relation to their working nature and unemployed person also travel from one place to the others due to the nature of not having fixed working place.

Our multivariable analysis showed that eating partially roasted meat was independent risk factors for contracting cholera infection. This may be due to the fact that some butcher house and slaughter house were affected by the cholera infection during the time of outbreak and may also be a problem of roasting temperature of the meat at food establishments.

Other risk factor identified in this outbreak investigation was being a male as independent risk factor for contracting cholera infection. This was likely because most of the time males use foods outside home which expose them to this infection. Other reason may also be due to high number of female controls and male cases were interviewed during the assessment because most of the time females were in their household during working day than males.

Regular hand washing with soap after defecation, disposing $\mathrm{HH}$ refuse at municipal site, treating water before drinking by aqua tabs and boiling methods at home were independent protective factors against contracting cholera infection. This findings were biologically plausible and supported with findings of cholera outbreak investigation in other settings [14-16].

Our multivariable analysis also shows that regular hand washing with soap after defecation was also other protective factor of contracting cholera infection. Similarly, the study conducted in India on cholera outbreak was also in line with this finding[15].

Disposing house hold refuse at municipal site was also independent protective factor for contracting cholera infection by $10.6 \%$. This finding is biologically plausible and likely because disposing house hold refuse at municipal site has less accessibility for the community to easily contaminate others.

Treating water before drinking by using aqua tabs and boiling methods at home were independent protective factors against contracting cholera infection. Likewise, the study conducted in Kadoma city of Zimbabwe [17] found that treating water by aqua tabs and boiling methods were protective factors against cholera infection. 
No significant association was found between cholera infection and eating food outside home, at restaurant. Likewise, the study conducted in Buea Health Districts of Cameroon in 2010 [16] found no significant association between eating food outside home and contracting of cholera infection.

\section{Conclusion}

Eating partially roasted meat, being a male, regular hand washing with soap after defecation, disposing house hold refuse at municipal site, treating water before drinking by aqua tabs and boiling were possible risk factors associated with the outbreak. Hence, water, sanitation and hygiene offices should strictly work on the hygiene and availability of safe water at all levels.

\section{Acknowledgments}

We would like to acknowledge Dr. Mamo Wubshet and Mr. Tewodros Getinet for their cooperation and comments on the write up of this out break investigation report. Our deepest gratitude also goes to the data collectors and study participants.

\section{Funding}

This outbreak investigation was supported by Ethiopian public health institute and Kirkos sub-city Health Department. The funders had no role in the study design, data collection, and analysis, decision to publish, or preparation of the manuscript.

\section{Availability of data and materials}

The datasets used and/or analyzed during the current outbreak investigation is available from the corresponding author (Tamiru Tadesse) on reasonable request.

\section{Authors' contributions}

TT identifies the gap, selected the title, designed the study, supervised data collection and data entry and made data analysis. The protocol development, reviewing of the data analysis, and edition was made by TT and BZ. BZ reviewed and approved the final manuscript.

\section{Authors' information}

TT: MPH in Field Epidemiology, Works at Border and Travel Health Directorate, Ethiopian Public Health Institute, Ethiopia. BZ: MSc in Biochemistry, PhD candidates, Works at Department of Biomedical sciences, Faculty of Medicine, Jimma University, Ethiopia.

\section{Ethics approval and consent to participate}

Approval and clearance were received from the Ethiopian Public Health Institute before the commencement of the study, by which the data collection tools with consent form, was approved. Then the formal letter was written to the selected four district health offices and official permission was obtained from each district health offices before the administration of the questionnaires. Considering the emergency situation at the time of the assessment, written consent was not obtained from the study participant. Verbal informed consent was obtained from each study participant and all information was kept confidential. Participation was voluntary and they were informed that all the data used was only for the scientific purpose and the Reponses of the outbreak. All participants were reassured that they can withdraw from the study at any time while interview. All findings and results presented were that of actual facts stated in the questionnaire and no false information or accusations were included.

\section{Consent for publication}

Not applicable because there is no identifiable image, videos or details relating to an individual person in our manuscript.

\section{Competing interests}

The authors declare that they have no competing interests.I

\section{References}

1. Ali M, Nelson AR, Lopez AL, Sack DA (2015) Updated global burden of cholera in endemic countries. PLoS Negl Trop Dis 9: e0003832. [Crossref]

2. Kirigia JM, Sambo LG, Yokouide A, Soumbey-Alley E, Muthuri LK, et al. (2009) Economic burden of cholera in the WHO African region. BMC Int Health Hum Rights 9: 8. [Crossref]

3. Assembly WH, Est S (2011) Releve epidemiologique hebdomadaire / Section d'hygiene du Secretariat de la Societe des Nations = Weekly epidemiological record $/$ Health Section of the Secretariat of the League of Nations (Wkly Epidemiol Rec) 86: 325-339.

4. Garcia E, Yactayo S, Nishino K, Millot V, Perea W, et al. (2016) Weekly epidemiological record Relevé épidémiologique hebdomadaire : 73-88.

5. Scrascia M, Pugliese N, Maimone F, Mohamud KA, Ali IA, et al. (2009) Cholera in Ethiopia in the 1990s: Epidemiologic patterns, clonal analysis, and antimicrobial resistance. Int J Med Microbiol 299: 367-372. [Crossref]

6. Hassen JK, Gizaw A, Mohamed S (2019) Determinants of pulmonary tuberculosis in public health facilities of Dire Dawa City, Eastern Ethiopia: Unmatched Case-control study. Int J Mycobacteriol 8: 118-123. [Crossref]

7. Zifan A (2016) Köppen climate classification. World Köppen Classif.

8. Dunkle SE, Mba-Jonas A, Loharikar A, Fouché B, Peck M, et al. (2011) Epidemic cholera in a crowded urban environment, Port-au-prince, Haiti. Emerg Infect Dis 17: 2143-2146. [Crossref]

9. Ethiopian Health and Nutrition Reaserch Institute E (2011) Guideline on CHOLERA OUTBREAK MANAGEMENT Ethiopia Ethiopian Health and Nutrition Research Institute Federal Democratic Republic of Ethiopia. [https://www.ephi.gov.et/images/ guidelines/national-cholera-guideline.pdf]

10. Beyene BB, Tumato M, Abera B, Maskai O, Luce R (2014) Epidemiology of Acute Watery Diarrhea Outbreak and Challenges of Control - Afar , Ethiopia , 2009. 1: 162-169.

11. Carrier, John W (2004) Cholera Outbreak.Assessing the outbreak response and improving prepardness. Br J Sociol 28: 520.

12. Ohene SA, Klenyuie W, Sarpeh M (2016) Assessment of the response to cholera outbreaks in two districts in Ghana. Infect Dis Poverty 5: 99. [Crossref]

13. Dalhat MM, Isa AN, Nguku P, Nasir SG, Urban K, et al. (2014) Descriptive characterization of the 2010 cholera outbreak in Nigeria. BMC Public Health 14: 1-7. [Crossref]

14. Ujjiga TTA, Wamala JF, Mogga JJH, Othwonh TO, Mutonga D, et al. (2015) Risk factors for sustained cholera transmission, Juba County, South Sudan, 2014. Emerg Infect Dis 21: 1849-1852. [Crossref]

15. Mridha P, Biswas AK, Ramakrishnan R, Murhekar MV (2011) The 2010 outbreak of cholera among workers of a jute mill in Kolkata, West Bengal, India. $J$ Health Popul Nutr 29: 9-13. [Crossref]

16. Nsagha DS, Atashili J, Fon PN, Tanue EA, Ayima CW, et al. (2015) Assessing the risk factors of cholera epidemic in the Buea Health District of Cameroon. BMC Public Health 15: 1-7.

17. Maponga BA, Chirundu D, Gombe NT, Tshimanga M, Shambira G, et al. (2013) Risk factors for contracting watery diarrhoea in Kadoma City, Zimbabwe, 2011: A case control study. BMC Infect Dis 13: 1-8. [Crossref]

Copyright: (C2019 Tadesse T. This is an open-access article distributed under the terms of the Creative Commons Attribution License, which permits unrestricted use, distribution, and reproduction in any medium, provided the original author and source are credited. 